\title{
Abstract PR09: Single cell phosphoproteomics identifies adaptive network dynamics of mTOR inhibitor resistance and defines effective combination therapy in glioblastoma
}

\author{
Young Shik Shin 1 , Wei Wei2, Beatrice Gini3 ${ }^{3}$ Paul Mischel3, and James Heath2
}

+ Author Affiliations

Abstracts: AACR Precision Medicine Series: Drug Sensitivity and Resistance: Improving Cancer Therapy; June 18-21, 2014; Orlando, FL

\begin{abstract}
Background: Resistance to single-agent targeted cancer therapy is almost universal. Resistance can occur when drug-resistant tumor cell subpopulations expand to drive recurrence in a process akin to Darwinian-type evolution under the selection pressure of the drug. An alternative resistance mechanism is the one in which cancer cells targeted by the inhibitor adapt to that drug, so as to maintain the signal flux through those networks that are required for tumor maintenance and growth. The main goal of this study is to identify the mechanisms of resistance in a targeted therapy by analyzing single cells and to provide a strategy to design a more effective therapy that suppresses resistance.
\end{abstract}

Methods: We investigated a clinically relevant model of acquired cancer drug resistance in glioblastoma (GBM). GBM39 is a human-derived primary GBM line that is maintained by serial transplantation in xenografts. GBM39 expresses high levels of the epidermal growth factor receptor (EGFR) variant(v)III oncogene, which sensitizes tumor cells to inhibitors of the mammalian target of rapamycin (mTOR). Therefore, we used this model to study acquired resistance to the mTOR kinase inhibitor CC214-2, which suppresses the proliferation of EGFRvIII expressing GBMs. We utilized a microfluidic device as a main tool that enables multiplex single cell proteomics. Relative to bulk assays, it can provide deeper insights into the signaling activity within a phosphoprotein signaling network, and how that activity is influenced by a drug. In addition to providing average protein levels, such assays also yield information on network signaling coordination that can be extracted from the variance for any given protein, as well as correlations between proteins. We determined the structure of the hyperactivated phosphoprotein signaling networks from statistical numbers of single cancer cells separated from the tumor for the untreated vehicle, during response to CC214-2, and after resistance to CC214-2.

Results: We find evidence that the tumors can rapidly adapt (within 2-3 days) to specific targeted inhibitors. The details of how the cells adapt, which are uniquely resolved by the single cell assays, can suggest effective therapy combinations. For a GBM murine model, tumor cells rapidly adapted to mTOR inhibition by activating ERK/Src signaling, suggesting that $\mathrm{mTOR}$ and ERK/Src signaling provided two independent druggable pathways for that tumor. Consonant with this hypothesis, we devised three therapeutic combinations and showed them to be highly effective in causing sustained clinical remission in vivo. A retrospective analysis revealed that mTOR signaling was a major driver of tumor growth in the untreated tumor, while ERK/Src signaling promoted resistance to mTOR inhibition. An additional study of a low passage, patient derived GBM cell line was also shown to exhibit rapid adaptation to single-agent targeted therapy in a manner that suggested effective combination therapies for cell killing. Finally, the analysis was shown to be feasible on a freshly resected patient GBM tumor.

Conclusions: The data and analysis presented here provide evidence that tumor cells can respond rapidly to a therapy and restores the growth characteristics temporarily disrupted by the therapy. This resistance mechanism is intrinsic; the same cells that respond to the therapy also adapt and develop resistance to it. For the GBM models explored here, a single cell proteomics analysis of the phosphoprotein signaling networks can resolve the independent signaling modes that drive tumor growth in both the untreated and drug resistant states. Such an analysis can be rapidly carried out using untreated tumor biopsies, and so may represent a new approach for guiding the selection of targeted combination therapies with low anticipated resistance.

Citation Format: Young Shik Shin, Wei Wei, Beatrice Gini, Paul Mischel, James Heath. 
Single cell phosphoproteomics identifies adaptive network dynamics of mTOR inhibitor resistance and defines effective combination therapy in glioblastoma. [abstract]. In: Proceedings of the AACR Precision Medicine Series: Drug Sensitivity and Resistance: Improving Cancer Therapy; Jun 18-21, 2014; Orlando, FL. Philadelphia (PA): AACR; Clin Cancer Res 2015;21(4 Suppl): Abstract nr PR09.

@2015 American Association for Cancer Research. 